\title{
Model Order Reduction for Networks of ODE and PDE Systems
}

\author{
Michael Hinze and Ulrich Matthes \\ Department of Mathematics, University of Hamburg, Bundesstr. \\ 55, 20146 Hamburg, Germany \\ michael.hinze@uni-hamburg.de \\ ulrich.matthes@math.uni-hamburg.de
}

\begin{abstract}
We propose a model order reduction (MOR) approach for networks containing simple and complex components. Simple components are modeled by linear ODE (and/or DAE) systems, while complex components are modeled by nonlinear PDE (and/or PDAE) systems. These systems are coupled through the network topology using the Kirchhoff laws. As application we consider MOR for electrical networks, where semiconductors form the complex components which are modeled by the transient drift-diffusion equations (DDEs). We sketch how proper orthogonal decomposition (POD) combined with discrete empirical interpolation (DEIM) and passivity-preserving balanced truncation methods for electrical circuits (PABTEC) can be used to reduce the dimension of the model. Furthermore we investigate residual-based sampling to construct reduced order models which are valid over a certain parameter range.
\end{abstract}

Keywords: Model Order Reduction, Parametrized Dynamical Systems, Drift-Diffusion Equations, Integrated Circuits.

AMS subject classifications: 93A30, 65B99, 65M60, 65M20.

\section{Introduction}

In this paper we propose a simulation-based MOR approach for the reduction of networks consisting of (many) simple and (only few) complex components. We assume that the simple and complex components are modeled by systems of linear ODEs (DAEs) and nonlinear PDEs (PDAEs), respectively, which are coupled through the network topology using the Kirchhoff laws.

As application we consider electrical networks where the simple components consist of resistors, capacitors, voltage sources, current sources, and inductors, and the complex components are formed by e.g. semi-conductors, see Figure 1 The overall system is then represented by a nonlinear partial differential algebraic equation (PDAE) system, see e.g. 38. In this paper we address the following issues:

1. construction of reduced order models for the complex components;

2. reduction of the complete network while retaining the structure of a network;

3. parametric MOR for complex components.

D. Hömberg and F. Tröltzsch (Eds.): CSMO 2011, IFIP AICT 391, pp. 92-101, 2013.

(C) IFIP International Federation for Information Processing 2013 


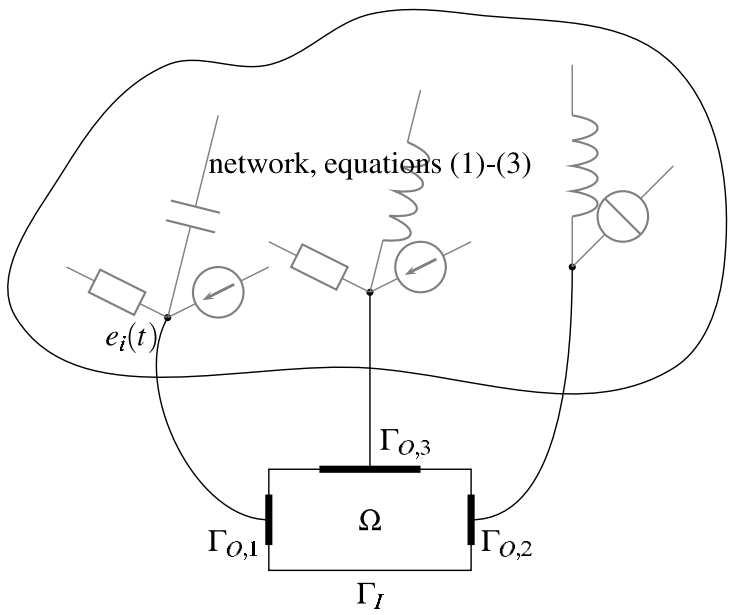

Fig. 1. Sketch of a coupled system with one semiconductor forming the complex component

\section{Example: Modeling of an Electrical Network}

In electrical networks resistors, capacitors, and inductors form the simple components which in general are modeled by linear ODEs. Complex components are given by e.g. semiconductors which are modeled by PDAE systems, see below. Considering additional voltage and current sources the overall network can be modeled by a PDAE which is obtained as follows. First the network containing only the simple components is modeled by a differential algebraic equation (DAE) which is obtained by a modified nodal analysis (MNA), including the Ohmic contacts $\Gamma_{O, k}$ of the semiconductors as network nodes, see Figure 1. Denoting by $e$ the node potentials and by $j_{L}, j_{V}$, and $j_{S}$ the currents of inductive, voltage source, and semiconductor branches, the DAE reads (see [812 19])

$$
\begin{aligned}
A_{C} \frac{d}{d t} q_{C}\left(A_{C}^{\top} e, t\right)+A_{R} g\left(A_{R}^{\top} e, t\right)+A_{L} j_{L}+A_{V} j_{V}+A_{S} j_{S} & =-A_{I} i_{s}(t), \\
\frac{d}{d t} \phi_{L}\left(j_{L}, t\right)-A_{L}^{\top} e & =0, \\
A_{V}^{\top} e & =v_{s}(t) .
\end{aligned}
$$

Here, the incidence matrix $A=\left[A_{R}, A_{C}, A_{L}, A_{V}, A_{S}, A_{I}\right]=\left(a_{i j}\right)$ represents the network topology, e.g. at each non mass node $i, a_{i j}=1$ if the branch $j$ leaves node $i$ and $a_{i j}=-1$ if the branch $j$ enters node $i$ and $a_{i j}=0$ elsewhere. The indices $R, C, L, V, S, I$ denote the capacitive, resistive, inductive, voltage source, semiconductor, and current source branches, respectively. In particular the matrix $A_{S}$ denotes the semiconductor incidence matrix. The vector valued functions $q_{C}, g$ and $\phi_{L}$ are continuously differentiable defining the voltage-current 
relations of the network components. The continuous vector valued functions $v_{s}$ and $i_{s}$ are the voltage and current sources. For details we refer to [10].

In a second step the semiconductors are modeled by PDAE systems, which are then coupled to the DAE of the network. Here we use the transient driftdiffusion equations as a continuous model for semiconductors, see e.g. 13] and the references cited there. Using the notation and scaling introduced there, we obtain the following scaled system of PDEs for the electrostatic potential $\psi(t, x)$, the electron and hole concentrations $n(t, x)$ and $p(t, x)$ and the current densities $J_{n}(t, x)$ and $J_{p}(t, x)$ :

$$
\begin{aligned}
\lambda \Delta \psi & =n-p-C, \\
-\partial_{t} n+\nu_{n} \operatorname{div} J_{n} & =R(n, p), \\
\partial_{t} p+\nu_{p} \operatorname{div} J_{p} & =-R(n, p), \\
J_{n} & =\nabla n-n \nabla \psi, \\
J_{p} & =-\nabla p-p \nabla \psi .
\end{aligned}
$$

Here $(t, x) \in[0, T] \times \Omega$ and $\Omega \subset \mathbb{R}^{d}$. The nonlinear function $R$ describes the rate of electron/hole recombination, $\lambda>0$ is the scaled Debye length, $\nu_{n}$ and $\nu_{p}$ are the scaled mobilities of electrons and holes. The temperature is assumed to be constant which leads to a constant thermal voltage $U_{T}$. The function $C$ is the time independent doping profile.

This system is supplemented with the boundary conditions

$$
\begin{aligned}
& \psi(t, x)=\psi_{b i}(x)+\left(A_{S}^{\top} e(t)\right)_{k}=U_{T} \log \left(\frac{\sqrt{C(x)^{2}+4 n_{i}^{2}}+C(x)}{2 n_{i}}\right)+\left(A_{S}^{\top} e(t)\right)_{k}, \\
& n(t, x)=\frac{1}{2}\left(\sqrt{C(x)^{2}+4 n_{i}^{2}}+C(x)\right), p(t, x)=\frac{1}{2}\left(\sqrt{C(x)^{2}+4 n_{i}^{2}}-C(x)\right),
\end{aligned}
$$

for $(t, x) \in[0, T] \times \Gamma_{O, k}$, where the potential of the nodes which are connected to a semiconductor interface enter in the boundary conditions for $\psi$. Here, $\psi_{b i}(x)$ denotes the build-in potential and $n_{i}$ the constant intrinsic concentration. All other parts of the boundary are isolation boundaries $\Gamma_{I}:=\Gamma \backslash \Gamma_{O}$, where $\nabla \psi \cdot \nu=0$, $J_{n} \cdot \nu=0$ and $J_{p} \cdot \nu=0$ holds. The semiconductor model (44)-(8) is coupled to the network through the semiconductor current vector $j_{S}$ with the components

$$
j_{S, k}=\int_{\Gamma_{O, k}}\left(J_{n}+J_{p}-\varepsilon \partial_{t} \nabla \psi\right) \cdot \nu d \sigma,
$$

where $\nu$ denotes the unit outward normal to the interface $\Gamma_{O, k}$. More details, including a precise description of the coupling, are given in [10]. The analytical and numerical analysis of PDAE systems of the presented form is subject to current research, see 37/16]19].

\section{Reduced Order Models for Complex Components}

We assume that every complex component is modeled by a time-dependent PDE or PDAE system which is amenable to a numerical treatment with Galerkin 
methods. After appropriate spatial discretization the method of lines then yields a large, nonlinear ODE system representing the spatially discrete complex component. This nonlinear ODE or DAE system now represents the complex component in the network. The reduction of the complex components is based on simulation-based MOR with POD. In this approach time snapshots of the complex components are extracted from snapshots of the simulation of the complete network. POD for the complex component then is performed using the extracted parts of the snapshots. In combination with DEIM [5] this now delivers low dimensional, nonlinear surrogate models for the complex components, see [9] for details.

Among other things it is an important feature of this reduction technique that it delivers distinct reduced order models for the same complex component at different locations in the network.

As example let us consider the rectifier network in Figure 2 (left). The POD basis functions of two identical semiconductors may be different due to different operating states of the semiconductors. Simulation results for this network are plotted in Figure 2 (right). Details of the implementation are sketched in Section 4. The distance between the linear spaces $U^{1}$ and $U^{2}$ which are spanned, e.g., by the POD-basis-functions $U_{\psi}^{1}$ associated to $\psi$ for the diode $S_{1}$ and $U_{\psi}^{2}$ associated to $\psi$ for the diode $S_{2}$ respectively, is measured by

$$
d\left(U^{1}, U^{2}\right):=\max _{\substack{u \in U^{1} \\\|u\|_{2}=1}} \min _{\substack{v \in U^{2} \\\|v\|_{2}=1}}\|u-v\|_{2} .
$$

Exploiting the orthonormality of the bases $U_{\psi}^{1}$ and $U_{\psi}^{2}$ and using a Lagrange framework, we find

$$
d\left(U^{1}, U^{2}\right)=\sqrt{2-2 \sqrt{\lambda}}
$$

where $\lambda$ is the smallest eigenvalue of the positive definite matrix $S S^{\top}$ with $S_{i j}=\left\langle u_{\psi, i}^{1}, u_{\psi, j}^{2}\right\rangle_{2}$. Here, $u_{\psi, i}^{1}$ denotes the i-th node in $U_{\psi}^{2}, u_{\psi, j}^{2}$ the $\mathrm{j}$-th node in $U_{\psi}^{2}$. The distances for the rectifier network are given in Table 1 . While the reduced model for the diodes $S_{1}$ and $S_{3}$ are almost equal, the reduced models for the diodes $S_{1}$ and $S_{2}$ are significantly different. Similar results are obtained for the reduction of the variables $n, p$, etc.

Table 1. Distances between reduced models in the rectifier network

\begin{tabular}{lll}
\hline$\Delta$ & $d\left(U^{1}, U^{2}\right)$ & $d\left(U^{1}, U^{3}\right)$ \\
\hline $10^{-4}$ & 0.61288 & $5.373 \cdot 10^{-8}$ \\
$10^{-5}$ & 0.50766 & $4.712 \cdot 10^{-8}$ \\
$10^{-6}$ & 0.45492 & $2.767 \cdot 10^{-7}$ \\
$10^{-7}$ & 0.54834 & $1.211 \cdot 10^{-6}$ \\
\hline
\end{tabular}



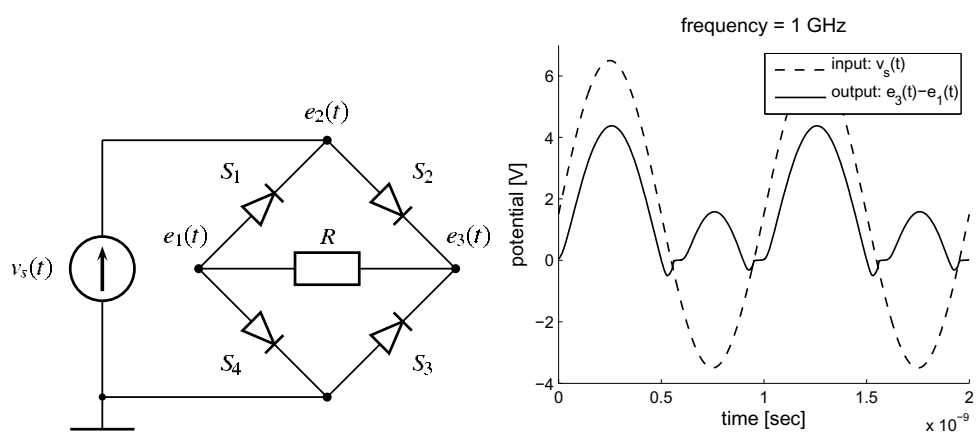

Fig. 2. Left: Rectifier network with 4 identical semiconductors. Right: Simulation results for the rectifier network. The input $v_{s}$ is sinusoidal with frequency $1 \mathrm{GHz}$ and offset $+1.5 \mathrm{~V}$. The time integration of the underlying nonlinear DAE system is performed with DASPK [414].

\section{Reduction of the Whole Network}

Let us assume that the overall network with simple and complex components now is represented by a nonlinear DAE system, where the linear part stems from the simple components, and the nonlinear part from the spatially-discrete complex components. The reduction for the complex components now is performed as in the previous section, whereas the linear part is approximated by a reduced order linear model of lower dimension. In the case of an electrical network the passivity preserving reduction method PABTEC [18] can be used to perform the reduction of the linear part of the network. Finally, the reduced order models, for the linear an the nonlinear part have to be recoupled appropriately, for details we refer to e.g. [17. To illustrate the performance of this approach we report on the numerical results obtained in [11] for an electrical network formed by an RC chain with one diode, see Figure 3 .

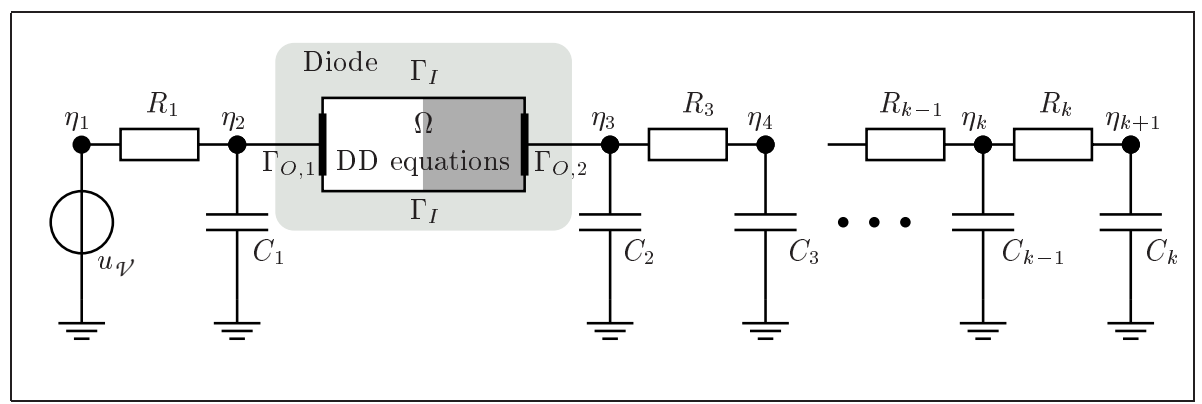

Fig. 3. RC chain with a diode 
For model reduction of the linear circuit equations we use the MATLAB Toolbox PABTEC [15]. The POD method is implemented in $\mathrm{C}++$ based on the FEM library deal.II [2] for discretizing the drift-diffusion equations. The obtained large and sparse nonlinear DAE system as well as the small and dense reducedorder model are integrated using the DASPK software package [4 based on a BDF method, where the nonlinear equations are solved using Newton's method. Furthermore, the direct sparse solver SuperLU [6] is employed for solving linear systems.

For the RC circuit with one diode in Figure 3 we use the input

$$
u(t)=u_{\mathcal{V}}(t)=10 \sin \left(2 \pi f_{0} t\right)^{4}
$$

with the frequency $f_{0}=10^{4} \mathrm{~Hz}$. The output of the system is $y(t)=-\imath_{\mathcal{V}}(t)$. We simulate the models over the fixed time horizon $\left[0, \frac{2.5}{f_{0}}\right]$. The linear resistors have the same resistance $R=2 \mathrm{k} \Omega$ and the linear capacitors have the same capacitance $C=0.02 \mu \mathrm{F}$.

We use the transient drift-diffusion equations to model the diode. For the parameters of the diode and the related scaling we refer to [11. In Table 2 we collect the numerical results for our reduction strategy. The outputs of the systems with the reduced network and POD-reduced diode are compared to the fully, spatially semidiscretized model with 7510 variables.

Here we construct a POD-reduced model for the diode based on a FE simulation with 500 nodes, where we apply DEIM for the reduction of the nonlinearity. The resulting reduced-order model for the diode is a dense nonlinear DAE of dimension 105 while the original spatially discrete model of the diode has dimension 6006. In Table 2 we summarize the results of the numerical simulations for the full nonlinear DAE system and the recoupled reduced system. The results demonstrate that the recoupling of the PABTEC reduced order model with the POD-MOR model for the semiconductor delivers an overall reduced-order model for the circuit-device system which allows significantly faster simulations (speedup-factor is about 20) while keeping the relative errors below $10 \%$.

In Figure 4 the evolution of the output currents is depicted for the full and the reduced systems. In addition, the evolutions of the output currents for the partially reduced systems (only reduction of the linear network, and only reduction of the diode) are shown.

Table 2. Statistics for model reduction of the coupled circuit-device system

\begin{tabular}{|c|c|c|c|c|}
\hline $\begin{array}{l}\text { network } \\
\text { (MNA } \\
\text { equations) }\end{array}$ & $\begin{array}{l}\text { diode } \\
\text { (DD } \\
\text { equations) }\end{array}$ & $\begin{array}{c}\text { dim. simul. } \\
\text { time }\end{array}$ & $\begin{array}{c}\text { Jacobian } \\
\text { evaluations }\end{array}$ & $\begin{array}{cc}\text { absolute } & \text { relative } \\
\text { error } & \text { error } \\
\|y-\hat{y}\|_{\mathbb{L}_{2}}\|y-\hat{y}\|_{\mathbb{L}_{2}} /\|y\|_{\mathbb{L}_{2}}\end{array}$ \\
\hline unred & unre & $751023.37 \mathrm{~s}$ & 20 & \\
\hline reduced & reduced & $1301.19 \mathrm{~s}$ & 11 & $2.954 \cdot 10^{-6} 1.000 \cdot 10^{-1}$ \\
\hline
\end{tabular}




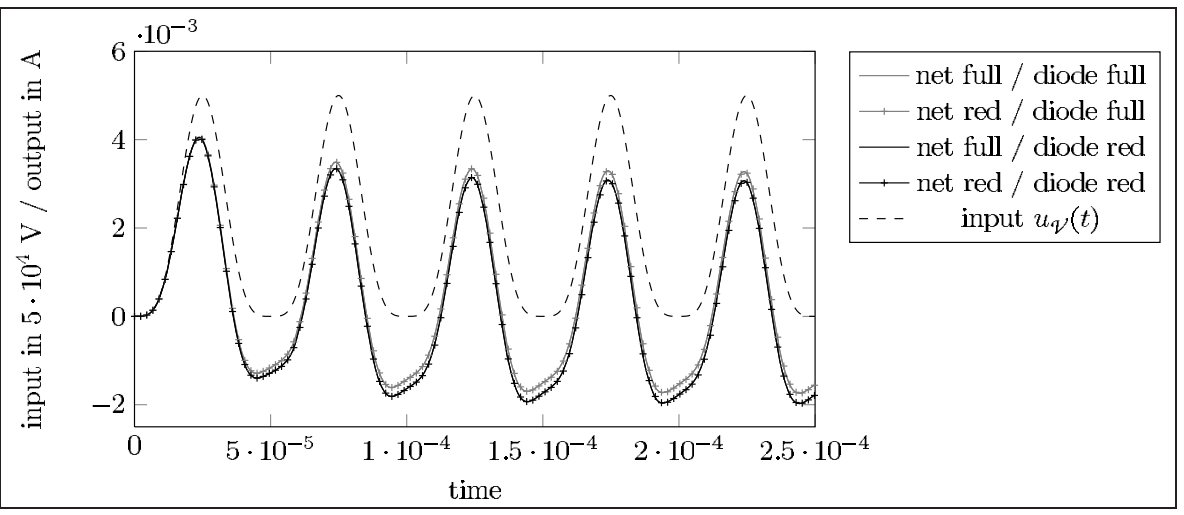

Fig. 4. Input voltage and output currents for different model reduction setups

\section{Parametric Model Order Reduction with Residual Based Sampling}

One major difficulty in simulation based MOR for complex components modeled by e.g. nonlinear PDE systems consists in the construction of reduced order models which are valid over a certain input parameter range, where the latter for electrical networks may be given by the input frequency. To obtain reduced order models for the complex components we propose residual based sampling which detects extreme parameters by evaluating the residual $\mathcal{R}$ of the reduced models over the parameter span. The greedy approach proposed in [13] then is used to enrich the simulation basis for the construction of a new reduced order model of the complex component, see Algorithm 1 .

We summarize our ideas in the following sampling algorithm, for details see [10]. Let $\mathcal{P}$ denote the parameter space and $\omega \in \mathcal{P}$ a parameter. Furthermore, let $\mathcal{R}\left(z^{P O D}(\omega, P)\right)$ denote the residual obtained by evaluation of the unreduced model at the solution of the reduced order model $z^{P O D}(\omega, P)$ based on snapshots taken on the parameter set $P \subset \mathcal{P}$.

\section{Algorithm 1 (Sampling)}

1. Select $\omega_{1} \in \mathcal{P}, P_{\text {test }} \subset \mathcal{P}$, tol $>0$, and set $k:=1, P_{1}:=\left\{\omega_{1}\right\}$. Simulate the unreduced model at $\omega_{1}$ and calculate the reduced model with POD basis functions $U^{1}$.

2. Calculate the residual $\left\|\mathcal{R}\left(z^{P O D}\left(\omega, P_{k}\right)\right)\right\|$ for all $\omega \in P_{\text {test }}$.

3. Check termination conditions, e.g.

$-\max _{\omega \in P_{\text {test }}}\left\|\mathcal{R}\left(z^{P O D}\left(\omega, P_{k}\right)\right)\right\|<$ tol, or

- no further reduction of residual, then STOP.

4. Calculate $\omega_{k+1}:=\arg \max _{\omega \in P_{\text {test }}}\left\|\mathcal{R}\left(z^{P O D}\left(\omega, P_{k}\right)\right)\right\|$. 
5. Simulate the unreduced model at $\omega_{k+1}$ and create a new reduced model with $P O D$ basis $U^{k+1}$ using also the already available information at $\omega_{1}, \ldots, \omega_{k}$.

6. Set $P_{k+1}:=P_{k} \cup\left\{\omega_{k+1}\right\}, k:=k+1$ and goto 3 .

The step 5 in Algorithm 1 can be executed in different ways. If offline time and offline memory requirements are not critical one may combine snapshots from all simulations of the full model and redo the model order reduction on the large snapshot ensemble. Otherwise a new reduced model at reference frequency $\omega_{k+1}$ may be constructed using the current POD-basis $\bar{U}$ and then perform an additional POD step on $\left(U_{k}, \bar{U}\right)$.

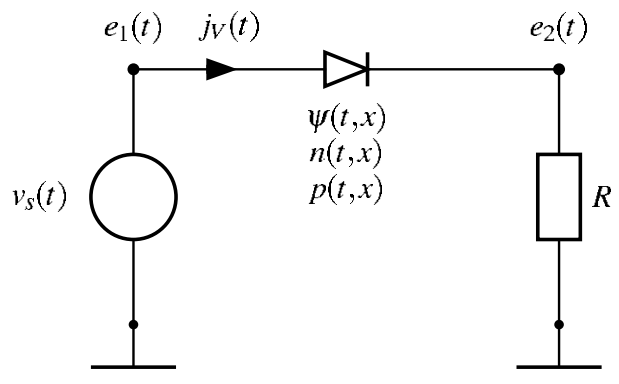

Fig. 5. Basic test circuit with one diode
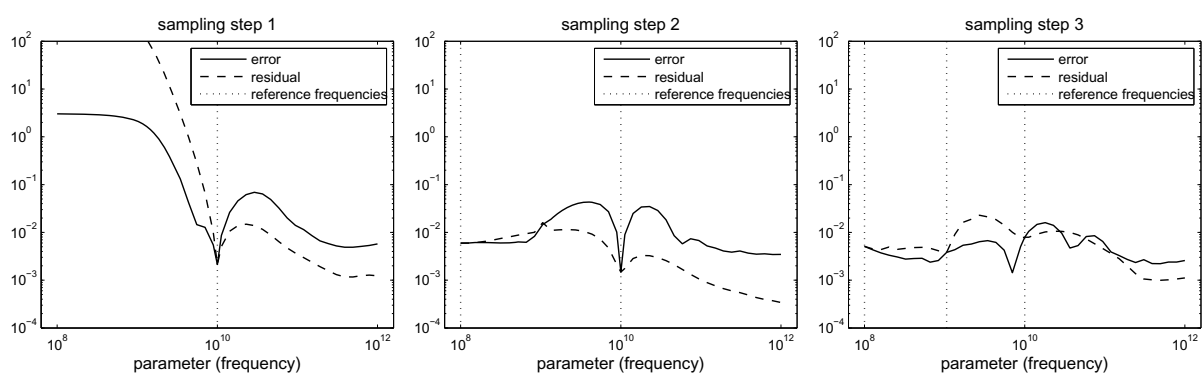

Fig. 6. Relative reduction error (solid line) and residual (dashed line) plotted over the frequency parameter space. The reduced model is based on simulations at the frequency parameter space. The reduced model is based on simulations at the reference frequencies $\omega_{1}:=10^{10} \mathrm{~Hz}$ (left), $\omega_{1}$ and $\omega_{2}:=10^{8} \mathrm{~Hz}$ (middle), and $\omega_{1}, \omega_{2}$, and $\omega_{3}:=1.0608 \cdot 10^{9} \mathrm{~Hz}$ (right). The reference frequencies are marked by vertical dotted lines.

To illustrate the performance of the sampling procedure we now apply Algorithm 1 to provide a reduced order model of the basic circuit shown in Figure 5 We choose the frequency of the input voltage $v_{s}$ as model parameter with parameter space $\mathcal{P}:=\left[10^{8}, 10^{12}\right] \mathrm{Hz}$. We initialize with a reduced model which 
Table 3. Performance of Algorithm 1

\begin{tabular}{llll}
\hline step $k$ & $\begin{array}{l}\text { reference parameters } \\
P_{k}\end{array}$ & $\begin{array}{l}\text { max. residual } \\
\text { (at frequency) }\end{array}$ & $\begin{array}{l}\text { max. relative error } \\
\text { (at frequency) }\end{array}$ \\
\hline 1 & $\left\{1.0000 \cdot 10^{10}\right\}$ & $9.9864 \cdot 10^{2}$ & $3.2189 \cdot 10^{0}$ \\
& & $\left(1.0000 \cdot 10^{8}\right)$ & $\left(1.0000 \cdot 10^{8}\right)$ \\
2 & $\left\{1.0000 \cdot 10^{8}\right.$, & $1.5982 \cdot 10^{-2}$ & $4.3567 \cdot 10^{-2}$ \\
& $\left.1.0000 \cdot 10^{10}\right\}$ & $\left(1.0608 \cdot 10^{9}\right)$ & $\left(3.4551 \cdot 10^{9}\right)$ \\
3 & $\left\{1.0000 \cdot 10^{8}\right.$, & $2.2829 \cdot 10^{-2}$ & $1.6225 \cdot 10^{-2}$ \\
& $1.0608 \cdot 10^{9}$, & $\left(2.7283 \cdot 10^{9}\right)$ & $\left(1.8047 \cdot 10^{10}\right)$ \\
& $\left.1.0000 \cdot 10^{10}\right\}$ & & \\
\hline
\end{tabular}

is constructed from the simulation of the full model at the reference frequency $\omega_{1}:=10^{10} \mathrm{~Hz}$. The number of POD basis functions $s$ is chosen such that the lack of information content $\Delta(s)$ is approximately $10^{-7}$. The relative error and the residual are plotted in Figure 6 (left). We observe that the residual admits a structure similar to that of the approximation error. Using Algorithm 1 the next additional reference frequency is $\omega_{2}:=10^{8} \mathrm{~Hz}$ since it maximizes the residual.

The next two iterations of the sampling algorithm are also depicted in Figure 6. Based on the residual in step 2 , one selects $\omega_{3}:=1.0608 \cdot 10^{9} \mathrm{~Hz}$ as the next reference frequency. Since no further reduction of the residual is achieved in step 3, the algorithm terminates. The maximal errors and residuals are given in Table 3. We note that in practical applications the error is not amenable over the whole parameter span. However the residual at least in the presented example seams to deliver a reliable indicator for the expected model error.

Acknowledgements. The work reported in this paper was supported by the German Federal Ministry of Education and Research (BMBF), grant no. 03HIPAE5. Responsibility for the contents of this publication rests with the authors.

\section{References}

1. Anile, A., Mascali, G., Romano, V.: Mathematical problems in semiconductor physics. Lectures given at the C. I. M. E. summer school, Cetraro, Italy, July 15-22, 1998. Lecture Notes in Mathematics. Springer, Berlin (2003)

2. Bangerth, W., Hartmann, R., Kanschat, G.: deal.II - a general-purpose objectoriented finite element library. ACM Trans. Math. Softw. 33(4) (2007)

3. Bodestedt, M., Tischendorf, C.: PDAE models of integrated circuits and index analysis. Math. Comput. Model. Dyn. Syst. 13(1), 1-17 (2007)

4. Brown, P., Hindmarsh, A., Petzold, A.: A description of DASPK: A solver for large-scale differential-algebraic systems. Tech. rep., Lawrence Livermore National Report UCRL (1992)

5. Chaturantabut, S., Sorensen, D.: Nonlinear model reduction via discrete empirical interpolation. SIAM J. Sci. Comput. 32(5), 2737-2764 (2010) 
6. Demmel, J.W., Eisenstat, S.C., Gilbert, J.R., Li, X.S., Liu, J.W.H.: A supernodal approach to sparse partial pivoting. SIAM J. Matrix Analysis and Applications 20(3), 720-755 (1999)

7. Günther, M.: Partielle differential-algebraische Systeme in der numerischen Zeitbereichsanalyse elektrischer Schaltungen. VDI Fortschritts-Berichte, Reihe 20, Rechnerunterstützte Verfahren, vol. 343 (2001)

8. Günther, M., Feldmann, U., ter Maten, J.: Modelling and discretization of circuit problems. In: Schilders, W.H.A., et al. (eds.) Handbook of Numerical Analysis. Special volume: Numerical methods in electromagnetics, vol. XIII, pp. 523-629. Elsevier/North Holland, Amsterdam (2005)

9. Hinze, M., Kunkel, M.: Discrete empirical interpolation in pod model order reduction of drift-diffusion equations in electrical networks. In: SCEE Proceedings 2010, Toulouse (2010)

10. Hinze, M., Kunkel, M.: Residual based sampling in POD model order reduction of drift-diffusion equations in parametrized electrical networks. Z. Angew. Math. Mech. 92, 91-104 (2012)

11. Hinze, M., Kunkel, M., Steinbrecher, A., Stykel, T.: Model order reduction of coupled circuit-device systems. Int. J. Numer. Model. (2012), doi:10.1002/jnm.840

12. Ho, C., Ruehli, A., Brennan, P.: The modified nodal approach to network analysis. IEEE Trans. Circuits Syst. 22, 504-509 (1975)

13. Patera, A., Rozza, G.: Reduced Basis Approximation and A Posteriori Error Estimation for Parametrized Partial Differential Equations. Version 1.0. Copyright MIT 2006-2007, to appear in (tentative rubric) MIT Pappalardo Graduate Monographs in Mechanical Engineering (2007)

14. Petzold, L.R.: A description of DASSL: A differential/algebraic system solver. IMACS Trans. Scientific Computing 1, 65-68 (1993)

15. Salih, H., Steinbrecher, A., Stykel, T.: MATLAB Toolbox PABTEC - A users guide. Technical Report, Institut für Mathematik, Technische Universität Berlin, Germany (2011)

16. Selva Soto, M., Tischendorf, C.: Numerical analysis of DAEs from coupled circuit and semiconductor simulation. Appl. Numer. Math. 53(2-4), 471-488 (2005)

17. Steinbrecher, A., Stykel, T.: Model order reduction of nonlinear circuit equations. Technical Report 2011/02, Institut für Mathematik, Technische Universität Berlin, Germany (2011)

18. Stykel, T., Reis, T.: The PABTEC algorithm for passivity-preserving model reduction of circuit equations. In: Proceedings of the 19th International Symposium on Mathematical Theory of Networks and Systems (MTNS 2010), July 5-9. ELTE, Budapest (2010) (paper 363)

19. Tischendorf, C.: Coupled Systems of Differential Algebraic and Partial Differential Equations in Circuit and Device Simulation. Habilitation thesis, HumboldtUniversity of Berlin (2003) 